\title{
噻吩甲酰硫脲衍生物的合成及其抑菌活性
}

\author{
程绎南*,a,b 靳文波 ${ }^{a}$ 谢桂英 ${ }^{a, b}$ 马艺超 赵艳芹 $a$ 李洪连 $*, a, b$ \\ $\left({ }^{a}\right.$ 河南农业大学植保学院 郑州 450002) \\ ( ${ }^{b}$ 新型农药创制与应用河南省重点实验室 郑州 450002)
}

\begin{abstract}
摘要 通过 3-颈基-2-丁酮与取代硅基丙炔酸甲酯的环合、酯的选择性水解、异硫氰化、胺化等步骤合成了 13 个未 报道的噻吩甲酰硫脲衍生物. 各噻吩甲酰硫艮衍生物的结构经 ${ }^{1} \mathrm{H} \mathrm{NMR} 、{ }^{13} \mathrm{C} \mathrm{NMR}$ 和元素分析的确证, 其对小麦全蚀 病、水稻纹枯病病原菌的抑菌活性经平皿法进行了测试. 结果表明, 大部分噻吩甲酰硫嫝衍生物对小麦全蚀病病原菌 具有一定抑制活性，其中 1-环丙基-3-(4,5-二甲基噻吩-3-甲酰基)硫脲(8c)抑菌活性表现较为突出，在 $10 \mathrm{mg} / \mathrm{L}$ 浓度下其 抑菌活性接近对照硅噻菌胺的水平. 结构分析表明噻吩-3-甲酰硫腿衍生物噻吩环 2 位官能团的大小对小麦全蚀病菌抑 制活性的影响不明显.
\end{abstract}

关键词 噻吩甲酰硫脲衍生物; 合成; 抑菌活性

\section{Synthesis of Thiophene Formyl Thiourea Derivatives and Fungicidal Activity}

\author{
Cheng, Yinan ${ }^{*, a, b} \quad$ Jin, Wenbo $^{a} \quad$ Xie, Guiying ${ }^{a, b} \quad$ Ma, Yichao \\ Zhao, Yanqin $^{a} \quad$ Li, Honglian*,a,b \\ ( ${ }^{a}$ Plant Protection College, Henan Agricultural University, Zhengzhou 450002) \\ ( ${ }^{b}$ Provincial Key Laboratory of the Discovery and Application of Novel Pesticide, Zhengzhou 450002)
}

\begin{abstract}
New thiophene formyl thiourea derivatives were synthesized via cycloaddition reaction of 3-mercaptobutan-2one and methyl 3-(substitutedsilyl)propiolate, selective hydrolysis of ester, isothiocyanatation and amination. Their chemical structures were confirmed by ${ }^{1} \mathrm{H}$ NMR, ${ }^{13} \mathrm{C}$ NMR and elemental analysis. The inhibitory activity against Gaeumannomyces graminis var. tritici and Rhizoctonia solani was evaluated in vitro by the plate method. The results indicated that most of thiophene formyl thiourea derivatives showed some inhibitory activity against gaeumannomyces graminis var. tritici. Compound 1-cyclopropyl-3-(4,5-dimethylthiophene-3-carbonyl)thiourea (8c) gave the best performance and its inhibitory activity was close to the control level of silthiopham in the concentration of $10 \mathrm{mg} / \mathrm{L}$. The structure analysis showed that the steric hindrance of functional group at the second position of the thiophene formyl thioureas had no obvious effect on the inhibitory activity against gaeumannomyces graminis var. tritici.
\end{abstract}

Keywords thiophene formyl thiourea derivatives; synthesis; inhibitory activity

硫䐂及酰基硫腿类衍生物具有广泛的生物活性，近 来引起人们的广泛关注. 一些芳基、杂芳基 $-N$-取代硫艮 衍生物具有较好的抑菌活性 ${ }^{[1]}$; 该类化合物还具有杀虫 活性, 如 1-特丁基-3-(2,6-二异丙基-4-苯氧基苯基)硫艮 ${ }^{[2]}$ 已经以商品名 “丁醚脲” 登记用于作物小菜蛾(Plutella xylostella)及红蜘蛛(Tetranychus cinnbarinus) 的防治. 硫 腺甲酰化后所形成的烷甲酰硫䐂和芳甲酰硫脲也具有
良好生物活性, 如烷甲酰硫脲衍生物对稗草 (Echinochloa crusgalli)、狗尾草(Setaira viridis)、马唐 (Digitaria sanguinalis)等杂草具有较好的抑制活性 ${ }^{[3]}$; 而 苯甲酰硫腿的核苷衍生物对水稻纹枯病 (Rhizoctonia solani)和芦笋茎枯病(Phomopsisasparagi bubak)等表现 出较好的抑菌活性 ${ }^{[4]}$; 苯甲酰硫䐂衍生物还对腿酶具有 较高的抑制活性等 ${ }^{[5]}$. 各种硫腿衍生物所表现出来的良

*E-mail: chyn212@aliyun.com; honglianli@sina.com

Received May 10, 2016; revised June 12, 2016; published online July 8, 2016.

Project supported by the Special Fund for Agro-scientific Research in the Public Interest (No. 201503112) and the Key Scientific Research Project of Colleges and Universities in Henan Province (No. 16A210006).

公益性行业(农业)科研专项基金(No. 201503112)、河南省高等学校重点科研(No. 16A210006)资助项目. 
好生物活性，为硫嫝活性化合物的设计提供了有用的工 具.

以噻吩为结构骨架的化合物在生物活性化合物家 族中也占有重要比重 ${ }^{[11]}$, 如 3-苯基-5-(噻吩-2)-1,2,4-噁 二唑已开发为杀虫剂 (tioxazafen) ${ }^{[6]}$, 而噻菌腈 $(\text { thicyofen })^{[7]}$ 、噻唑菌胺 (ethaboxam $)^{[8]}$ 、硅噻菌胺 (silthiopham) ${ }^{[9]}$ 、吡噻菌胺(penthiopyrad) ${ }^{[10]}$ 等已分别开发 为农用杀菌剂. 近来我们也致力于噻吩活性化合物的合 成与开发 ${ }^{[11,12]}$, 发现一些噻吩甲酰胺衍生物对小麦全蚀 病具有优异的抑制活性, 然而有关噻吩甲酰基硫腿衍生 物的结构与活性研究还涉及的很少. 作为该工作的一部 分, 我们拟设计合成一系列噻吩甲酰基硫艮类衍生物, 研究它们的结构与活性关系, 以期获得对小麦全蚀病具 有较高抑制活性的新先导化合物.

为此, 我们按照 Scheme 1 所示路线合成了 13 个未 见有报道的噻吩甲酰基硫腿衍生物, 来考察硫腿胺基结 构及噻吩 2 位官能团的变化对其生物活性的影响.
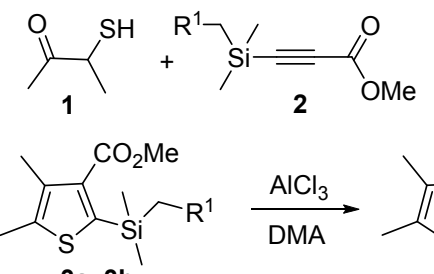

$\underset{\text { (2) acetic anhydride }}{\stackrel{\text { (1) } 0.1 \text { equiv. } t \text {-BuOK }}{\longrightarrow}}$

$3 a, 3 b$

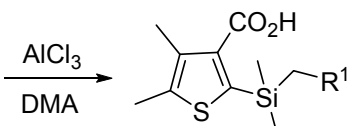

$\underset{\text { (2) } \mathrm{KSCN}}{\stackrel{(1) \mathrm{SOCl}_{2}}{\longrightarrow}}$<smiles>[R]C[Si](C)(C)c1sc(C)c(C)c1C(=O)NS(C)(=O)=O</smiles>

5a, 5b

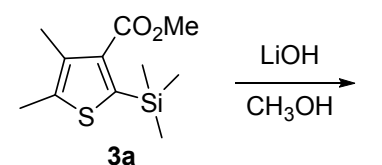

$3 a$

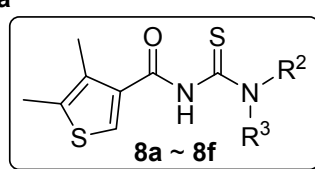

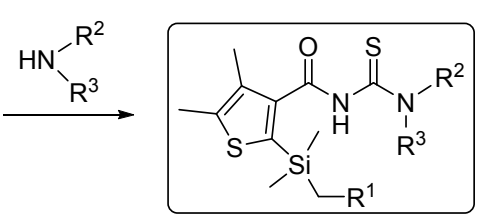

$6 \mathrm{a} \sim 6 \mathrm{~g}$
(1) $\mathrm{SOCl}_{2} / \mathrm{KSCN}$

(2) $\mathrm{HN}_{-}^{-\mathrm{R}^{2}}$

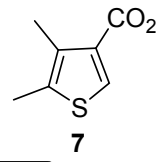

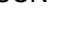

.

6a: $\mathrm{R}^{1}=\mathrm{H}, \mathrm{R}^{2}=$ allyl, $\mathrm{R}^{3}=\mathrm{H} ; \mathbf{6 b}: \mathrm{R}^{1}=\mathrm{H}, \mathrm{R}^{2}=i-\mathrm{Pr}, \mathrm{R}^{3}=\mathrm{H} ; \mathbf{6 c}: \mathrm{R}^{1}=\mathrm{H}$, $\mathrm{R}^{2}=$ cyclopropyl, $\mathrm{R}^{3}=\mathrm{H} ; \mathbf{6 d}: \mathrm{R}^{1}=\mathrm{H}, \mathrm{R}^{2}=$ cyclopropylmethyl, $\mathrm{R}^{3}=\mathrm{H}$; 6e: $R^{1}=H, R^{2}=3$-chlorobenzyl, $R^{3}=H ; 6 f: R^{1}=E t, R^{2}=E t, R^{3}=H$; 6g: $\mathrm{R}^{1}=\mathrm{Cl}, \mathrm{R}^{2}=$ allyl, $\mathrm{R}^{3}=\mathrm{H} ; \mathbf{8 a}: \mathrm{R}^{2}=$ allyl, $\mathrm{R}^{3}=\mathrm{H} ; \mathbf{8 b}: \mathrm{R}^{2}=i-\mathrm{Pr}, \mathrm{R}^{3}$ $=\mathrm{H} ; \mathbf{8 c}: \mathrm{R}^{2}=$ cyclopropyl, $\mathrm{R}^{3}=\mathrm{H} ; \mathbf{8 d}: \mathrm{R}^{2}=$ cyclopropylmethyl, $\mathrm{R}^{3}=$ $\mathrm{H} ; \mathbf{8 e}: \mathrm{R}^{2}=3$-chlorobenzyl, $\mathrm{R}^{3}=\mathrm{H} ; \mathbf{8 f}: \mathrm{R}^{2}=\mathrm{Et}, \mathrm{R}^{3}=\mathrm{Et}$

图式 1 取代的噻吩甲酰基硫脲的合成路线

Scheme 1 Synthetic routes of target compounds

\section{1 结果与讨论}

\subsection{4,5-二甲基-2-硅基噻吩-3-甲酸甲酯的合成}

目标化合物的合成过程中, 噻吩环上硅官能团的引

入具有较大的挑战. 通常硅官能团的引入是在丁基 锂 ${ }^{[13,14]}$ 或过渡金属 ${ }^{[15 ~ 17]}$ 的催化下实现的. 这些方法具 有反应条件苛刻、成本高的缺点. 我们前期的研究开发 了在甲醇钠的促进下由 $a$-颈基酮与 3-三甲基硅基丙炔 酸酯的环合直接实现在噻吩环上引入硅基的方法 ${ }^{[12]}$, 然而，甲醇钠的使用是等当量的. 作为该研究的进一步 深入，我们开发了以叔丁醇钾为催化剂的更加高效、绿 色的合成方法. 催化剂篮选数据如表 1 所示.

表 1 3-三甲基硅基丙炔酸甲酯与 3-颈基-2-丁酮的催化环合 ${ }^{a}$ Table 1 Cyclization reaction of methyl trimethylsilylpropiolate and 3-mercapto-2-butanone

\begin{tabular}{|c|c|c|c|c|c|c|}
\hline Entry & Promoter & $\begin{array}{l}\text { Load/ } \\
\text { equiv. }\end{array}$ & $T /{ }^{\circ} \mathrm{C}$ & Time/h & Solvent & Yield $^{b} / \%$ \\
\hline 1 & $\mathrm{FeCl}_{3} \cdot 6 \mathrm{H}_{2} \mathrm{O}$ & 0.1 & r.t. $\sim 110$ & 10 & DMSO & 0 \\
\hline 2 & $\mathrm{FeCl}_{3}$ & 0.1 & r.t. $\sim 110$ & 10 & DMSO & 0 \\
\hline 3 & $\mathrm{CuCl}_{2}$ & 0.1 & r.t. $\sim 110$ & 10 & DMSO & 0 \\
\hline 4 & $\mathrm{CuCl}$ & 0.1 & r.t. $\sim 110$ & 10 & DMSO & 0 \\
\hline 5 & $\mathrm{CuO}$ & 0.1 & r.t. $\sim 110$ & 10 & DMSO & 0 \\
\hline 6 & $\mathrm{Ph}_{3} \mathrm{P}$ & 0.1 & r.t. $\sim 110$ & 10 & Toluene & 0 \\
\hline 7 & DABCO & 0.1 & r.t. $\sim 110$ & 10 & Toluene & 0 \\
\hline 8 & $\mathrm{NEt}_{3}$ & 0.1 & r.t. $\sim 110$ & 10 & Toluene & 0 \\
\hline 9 & $t$-BuOK & 0.1 & r.t. & 3 & Toluene & 87 \\
\hline 10 & $t$-BuOK & 0.5 & r.t. & 3 & Toluene & 59 \\
\hline 11 & $t$-BuOK & 0.05 & r.t. & 3 & Toluene & 67 \\
\hline 12 & $t$-BuOK & 0.1 & r.t. & 10 & Toluene & 72 \\
\hline 13 & $t$-BuOK & 0.1 & r.t. & 3 & DMF & 33 \\
\hline 14 & $t$-BuOK & 0.1 & r.t. & 3 & DMSO & 13 \\
\hline
\end{tabular}

${ }^{a} 1$ equiv. of acetic anhydride was used in all reactions. ${ }^{b}$ Isolated yields based on methyl trimethylsilylpropiolate.

为了探索更加高效、绿色的合成方法, 我们参考了 $\mathrm{FeCl}_{3}{ }^{[18]}$ 和 $\mathrm{Cu}$ 盐 ${ }^{[19]}$ 催化的 $a$-羟基酮与取代的丙炔酸酯 环合的方法, 但没有获得成功(表 1, Entries 1 5). 我们 还尝试了以三苯基磷 $\left(\mathrm{Ph}_{3} \mathrm{P}\right)^{[20]}$ 、三乙烯二胺 (DAB$\mathrm{CO})^{[21]}$ 、三乙胺 ${ }^{[22]}$ 等为催化剂的反应体系. 然而, 即使 将这些催化剂的用量增加到反应物的等物质的量, 对反 应也没有提供满意的促进效果. 有文献报道了以叔丁醇 钾 ${ }^{[23]}$ 为催化剂合成噻吩羰基酸酯的方法, 尽管相应产 品的收率只有 $20 \% \sim 58 \%$. 我们还是考察了其对该反应 的催化性能. 当在 0.1 equiv. 的叔丁醇钾存在下, 室温下 反应 $3 \mathrm{~h}$ 获得了 $87 \%$ 的收率(Entry 9). 当催化剂的量增 加到 0.5 equiv.时，收率没有增加反而降到 59\% (Entry 10). 分析表明原料转化完全, 但伴有少量脱硅烷化的 4,5-二甲基噻吩-3-甲酸甲酯生成. 可能是形成的产品不 能承受较多叔丁醇钾存在时形成的强碱环境而发生产 品的脱硅烷化所致; 相对地, 催化量的叔丁醇钾对产品 的脱硅烷化作用受到较大的抑制. 进一步降低催化剂用 
量, 发现产率降低、原料转化不完全(Entry 11). 于是我 们进一步考察反应时间对反应的影响, 维持温度和催化 剂的量不变, 把反应时间进一步延长到 $10 \mathrm{~h}$, 收率没有 得到进一步提高(Entry 12). 接着考察溶剂对反应的影 响，当以强极性非质子溶剂DMSO、DMF为反应媒介时， 叔丁醇钾催化活性较低, 只得到不足 $35 \%$ 的收率 (Entries 13，14). 最终优化的反应条件为: 以甲苯为溶 剂, 在 0.1 equiv.叔丁醇钾的存在下, 在室温反应 $3 \mathrm{~h}$. 该 方法适用于 3-氯甲基二甲基硅基丙炔酸甲酯与 3-颈基 -2-丁酮的环合，收率 78\%.

\subsection{4,5-二甲基-2-取代噻吩-3-甲酸的合成}

4,5-二甲基-2-取代噻吩-3-甲酰基硫嫝衍生物的合 成是通过 4,5-二甲基-2-取代噻吩-3-甲酸的酰化、异硫氰 化、胺化来实现的. 我们利用 4,5-二甲基-2-硅基噻吩- 3甲酸甲酯中取代硅基对不同水解条件的敏感性差异, 选 用三氯化铝 $\left(\mathrm{AlCl}_{3}\right) / N, N$-二甲基苯胺(DMA) $(V: V=3$ : 4)水解体系, 选择性地得到 4,5-二甲基-2-取代硅基噻吩3-甲酸 ${ }^{[12]}$; 选用氢氧化锂 $(\mathrm{LiOH}) /$ 甲醇 $\left(\mathrm{CH}_{3} \mathrm{OH}\right) /$ 水 $\left(\mathrm{H}_{2} \mathrm{O}\right)$ 水解体系, 得到脱硅烷化的 4,5-二甲基噻吩-3-甲酸. 进 而分别通过酰化、异硫氧化、胺化等步骤方便地得到相 应的硫腿衍生物。

\section{3 噻吩甲酰基硫腿衍生物抑菌活性}

为了评估噻吩甲酰基硫腿衍生物的抑菌活性, 我们 采用平皿法 ${ }^{[24]}$ 以菌丝生长速率来测试所合成新化合物 $6 \mathrm{a} \sim 6 \mathrm{~g}$ 和 8a $\sim 8 \mathrm{f}$ 对小麦全蚀病病原菌的离体抑制活性, 测试结果如表 2 所示. 在所合成的两个系列噻吩甲酰基 硫腿衍生物中, 除 $8 \mathrm{f}$ 外, 大部分化合物对小麦全蚀病 病原菌都表现出一定的抑制活性, $6 \mathrm{~b} 、 6 \mathrm{~g} 、 8 \mathrm{a} \sim 8 \mathrm{~d}$ 在 10 $\mathrm{mg} / \mathrm{L}$ 浓度下抑制活性表现较好, 其中尤以 $8 \mathrm{c}$ 抑菌活性 表现更为突出, 在 $10 \mathrm{mg} / \mathrm{L}$ 浓度下其抑菌活性接近对照 硅噻菌胺的水平. 初步结构分析可以看出, 噻吩-3-甲酰 基硫脲衍生物 2 位官能团的大小对活性的影响并不明 显, 这与我们曾经报道的噻吩-3-甲酰胺衍生物 2 位官能 团的大小对活性的影响显著 ${ }^{[11]}$ 的现象存在明显差异, 这 种差异可能是由于该两类化合物发挥抑菌作用的机制 不同所产生的. 除此之外, 我们还考察了噻吩甲酰基硫 腿衍生物对水稻纹枯病病菌的抑制活性, 遗憾的是在所 设计浓度 $(10 、 1 、 0.1 \mathrm{mg} / \mathrm{L})$ 下均没有表现出抑制活性.

\section{2 结论}

以 3-颈基-2-丁酮和 3-取代硅基丙炔酸甲酯为起始 原料, 以叔丁醇钾为催化剂通过直接环合反应合成了 4,5-二甲基-2-硅基噻吩-3-甲酸甲酯衍生物. 4,5-二甲基-
表 2 噻吩甲酰硫脲衍生物对小麦全蚀病病原菌的离体抑制 活性 $^{a}$

Table 2 Inhibitory activity of thiophene formyl thiourea derivatives against gaeumannomyces graminis var. tritici

\begin{tabular}{cccc}
\hline \multirow{2}{*}{ Compd. } & \multicolumn{3}{c}{ Percent inhibition/\% (In vitro) } \\
\cline { 2 - 4 } & $10 \mathrm{mg} / \mathrm{L}$ & $1 \mathrm{mg} / \mathrm{L}$ & $0.1 \mathrm{mg} / \mathrm{L}$ \\
\hline $\mathbf{6 a}$ & 15 & 11 & 2 \\
$\mathbf{6 b}$ & 36 & 19 & 9 \\
$\mathbf{6 c}$ & 21 & 14 & 13 \\
$\mathbf{6 d}$ & 23 & 22 & 14 \\
$\mathbf{6 e}$ & 27 & 25 & 24 \\
$\mathbf{6 f}$ & 25 & 20 & 18 \\
$\mathbf{6 g}$ & 37 & 23 & 15 \\
$\mathbf{8 a}$ & 36 & - & - \\
$\mathbf{8 b}$ & 48 & 20 & - \\
$\mathbf{8 c}$ & 72 & 11 & - \\
$\mathbf{8 d}$ & 40 & 10 & - \\
$\mathbf{8 e}$ & 20 & 12 & - \\
$\mathbf{8 f}$ & - & - & - \\
Silthiopham & 77 & 68 & 62 \\
\hline a “-”No activity. & & &
\end{tabular}

2-硅基塞吩-3-甲酸甲酯衍生物分别通过三氯化铝 $/ N, N$ 二甲基苯胺体系和氢氧化锂/甲醇/水体系的选择性水解, 分别得到 2-硅基噻吩-3-甲酸及 2 位无取代基的噻吩-3甲酸衍生物, 进而高效地合成得到了 13 个噻吩-3-甲酰 硫腿衍生物. 活性测试表明, 除 $8 \mathbf{f}$ 外大部分化合物对小 麦全蚀病病原菌均表现出一定的抑制活性，在 $10 \mathrm{mg} / \mathrm{L}$ 浓度下, $8 \mathrm{c}$ 的抑菌活性接近或达到了对照硅噻菌胺的活 性水平. 初步结构活性分析发现, 噻吩-3-甲酰硫腿衍生 物 2 位官能团的大小对小麦全蚀病菌的抑制活性没有明 显影响. 这些发现为抑菌活性化合物的进一步结构设计 提供了参考.

\section{3 实验部分}

\section{1 仪器与试剂}

GC 或 HPLC 用于反应的检测和分析; ${ }^{1} \mathrm{H} N M R 、{ }^{13} \mathrm{C}$ NMR 用 Agilent-NMR-vnmrs 400 核磁共振仪 $\left(\mathrm{CDCl}_{3}\right.$ 为 溶剂, TMS 为内标); 质谱用 Bruker micrOTOF-Q II 质谱 仪; 元素分析用德国 Elememtar Vario-III 型元素分析仪; 熔点用 WRS-1A 数字熔点仪; 病原菌培养用 HPG-280H 人工气候箱. 所用进口或国产市售分析纯或化学纯试剂 未经纯化直接使用.

\section{2 病原菌菌株}

供试的小麦全蚀病菌(Gaeumannomyces graminis var. tritici) 由河南农业大学植物保护学院小麦病害研究 室提供; 水稻纹枯病菌(Rhizoctonia solani)由河南农业 大学植物保护学院水稻病害研究室提供. 


\section{3 中间体及目标化合物的合成}

3.3.14,5-二甲基-2-取代硅基噻吩-3-甲酸甲酯(3a, 3b)的合成

将 3-颈基-2-丁酮(5.72 g, $55 \mathrm{mmol}$ )投入盛有 $35 \mathrm{~mL}$ 甲苯的 $100 \mathrm{~mL}$ 园底反应瓶中, 然后向反应瓶中加入 $3-$ 取代硅基丙炔酸甲酯 $(50 \mathrm{mmol})$. 在搅拌下加入叔丁醇 钾 $(0.56 \mathrm{~g}, 5 \mathrm{mmol})$, 氮气保护下, 室温反应, 气相色谱检 测, 直到反应完全, 约需 $3 \mathrm{~h}$. 反应完全后, 再向反应体 系中加入乙酸酐 $(5.6 \mathrm{~g}, 50 \mathrm{mmol})$ 后, 加热到 $80{ }^{\circ} \mathrm{C}$ 维持 3 h. 然后脱出大部分的溶剂, 并用质量分数为 $10 \%$ 碳酸 钠水溶液洗涤, 二氯甲烷萃取, 无水硫酸钠干燥. 干燥 后的有机相先在负压下脱出溶剂, 然后再用硅胶柱层析 分离, 得到相应的4,5-二甲基-2-取代硅基噻吩-3-甲酸甲 酯.

\subsubsection{4,5-二甲基-2-硅基噻吩-3-甲酸 $(\mathbf{4 a}, \mathbf{4 b})$ 的合成}

向盛有 $15 \mathrm{~mL}$ 水和 $15 \mathrm{~mL}$ 甲醇的 $100 \mathrm{~mL}$ 圆底烧瓶 中投入 $1.3 \mathrm{~g}(55 \mathrm{mmol})$ 氢氧化锂和 $12.1 \mathrm{~g}(50 \mathrm{mmol})$ 的 4,5-二甲基-2-三甲基硅基噻吩-3-甲酸甲酯, 加热回流 3 h. 负压下蒸出溶剂, 残留物用水溶解、盐酸酸化 $(\mathrm{pH}<$ 2 )、过滤、滤饼干燥得 4,5-二甲基噻吩-3-甲酸(7.2 g), 收 率 $92 \%$.

4,5-二甲基-2-硅基噻吩-3-甲酸按照文献[12]方法选 择性水解得到.

3.3.3 $N$-取代-4,5-二甲基-2-取代噻吩-3-甲酰硫脲 (6a $\sim 6 \mathrm{~g}, 8 \mathrm{a} \sim 8 \mathrm{8f})$ 的合成

将(5 mmol) 4,5-二甲基-2-取代噻吩-3-甲酸投入盛 有 $10 \mathrm{~mL}$ 甲苯、带有回流和气体吸收装置的三口反应瓶 中, 在回流温度下缓慢加入 $708 \mathrm{mg}(6 \mathrm{mmol})$ 的氯化亚 砜, 加完后继续回流反应 $2 \mathrm{~h}$, 负压脱出溶剂和过量的 氯化亚砜后, 向反应体系中补加 $10 \mathrm{~mL}$ 乙腈, 再边搅拌 边加入 $534 \mathrm{mg}(5.5 \mathrm{mmol})$ 硫氰化钾 $(\mathrm{KSCN})$, 回流温度 下反应 $2 \mathrm{~h}$ 后降温、过滤. 滤液转至反应瓶中, 分别加入 各种取代胺 $(5.5 \mathrm{mmol})$, 继续回流反应 $2 \mathrm{~h}$. 反应液浓 缩、水洗、硅胶柱分离, 得浅黄色固体产品.

1-烯丙基-3-(4,5-二甲基-2-三甲基硅基噻吩-3-甲酰 基)硫脲(6a)：淡黄色固体，收率 80\%. m.p. 106.5 $108.7{ }^{\circ} \mathrm{C} ;{ }^{1} \mathrm{H}$ NMR (400 MHz, $\mathrm{CDCl}_{3}$ ) $\delta: 10.67$ (br s, $1 \mathrm{H}$, $\mathrm{NH}), 8.60($ br s, $1 \mathrm{H}, \mathrm{NH}), 6.02 \sim 5.94\left(\mathrm{~m}, 1 \mathrm{H}, \mathrm{NCH}_{2-}\right.$ $\mathrm{CH}=), 5.35 \sim 5.26\left(\mathrm{dd}, J=16.8,10.4 \mathrm{~Hz}, 2 \mathrm{H},=\mathrm{CH}_{2}\right)$, $4.39 \sim 4.36\left(\mathrm{dd}, J=5.6,5.2 \mathrm{~Hz}, 2 \mathrm{H}, \mathrm{NHCH}_{2}\right), 2.37$ (s, 3H, $\left.\mathrm{ArCH}_{3}\right), 2.23$ (s, 3H, $\left.\mathrm{ArCH}_{3}\right), 0.37$ (s, 9H, $\left.\mathrm{SiCH}_{3}\right) ;{ }^{13} \mathrm{C}$ NMR (100 MHz, $\left.\mathrm{CDCl}_{3}\right) \delta: 179.77,166.91,141.79$, $140.48,140.32$, 132.96, 131.91, 117.52, 47.89, 13.35, 12.85, - 0.04. Anal. calcd for $\mathrm{C}_{14} \mathrm{H}_{22} \mathrm{~N}_{2} \mathrm{OS}_{2} \mathrm{Si}$ : C 51.49, H 6.79, N 8.58, S 19.64; found C 51.34, H 6.81, N 8.55, S
19.60.

1-(4,5-二甲基-2-三甲基硅基噻吩-3-甲酰基)-3-异丙 基硫脲(6b): 淡黄色固体，收率 76\%. m.p. 115.8 $116.5{ }^{\circ} \mathrm{C}$; ${ }^{1} \mathrm{H}$ NMR (400 MHz, $\mathrm{CDCl}_{3}$ ) $\delta$ : 10.47 (br s, $1 \mathrm{H}$, $\mathrm{NH}), 8.47$ (br s, 1H, NH), $4.58 \sim 4.53(\mathrm{~m}, 1 \mathrm{H}, \mathrm{NCH}), 2.37$ $\left(\mathrm{s}, 3 \mathrm{H}, \mathrm{ArCH}_{3}\right), 2.22\left(\mathrm{~s}, 3 \mathrm{H}, \mathrm{ArCH}_{3}\right), 1.37 \sim 1.35$ (d, $J=6.4$ $\left.\mathrm{Hz}, 6 \mathrm{H}, \mathrm{NCHCH}_{3}\right), 0.36\left(\mathrm{~s}, 9 \mathrm{H}, \mathrm{SiCH}_{3}\right) ;{ }^{13} \mathrm{C} \mathrm{NMR}(100$ $\left.\mathrm{MHz} \mathrm{CDCl}_{3}\right) \delta: 178.14,166.97,142.05,140.38,139.91$, $132.99,47.74,21.74,13.32,12.79,0.07$. Anal. calcd for $\mathrm{C}_{14} \mathrm{H}_{24} \mathrm{~N}_{2} \mathrm{OS}_{2} \mathrm{Si}$ : C 51.18, H 7.36, N 8.53, S 19.52; found C 51.09, H 7.39, N 8.48, S 19.43.

1-环丙基-3-(4,5-二甲基-2-三甲基硅基噻吩-3-甲酰 基)硫脲(6c)：淡黄色固体，收率 65\%. m.p. 133.4 $134.1{ }^{\circ} \mathrm{C} ;{ }^{1} \mathrm{H}$ NMR (400 MHz, $\mathrm{CDCl}_{3}$ ) $\delta: 10.53$ (br s, $1 \mathrm{H}$, $\mathrm{NH}), 8.55$ (br s, 1H, NH), 3.24 3.20 (m, 1H, NCH), 2.36 $\left(\mathrm{s}, 3 \mathrm{H}, \mathrm{CCH}_{3}\right), 2.21\left(\mathrm{~s}, 3 \mathrm{H}, \mathrm{CCH}_{3}\right), 0.99 \sim 0.95(\mathrm{~m}, 2 \mathrm{H}$, $\mathrm{CHCH}), 0.79$ (s, 2H, CHCH), $0.35\left(\mathrm{~s}, 9 \mathrm{H}, \mathrm{SiCH}_{3}\right) ;{ }^{13} \mathrm{C}$ NMR $\left(100 \mathrm{MHz}, \mathrm{CDCl}_{3}\right) \delta: 181.04,166.86,141.87$, $140.42,140.12,133.00,28.06,13.31,12.80,7.29$, -0.05 . Anal. calcd for $\mathrm{C}_{14} \mathrm{H}_{22} \mathrm{~N}_{2} \mathrm{OS}_{2} \mathrm{Si}$ : C 51.49, H 6.79, N 8.58, S 19.64; found C 51.40, H 6.77, N 8.54, S 19.58.

1-环丙基甲基-3-(4,5-二甲基-2-三甲基硅基噻吩-3甲酰基)硫嫝(6d): 淡黄色固体，收率 73\%. m.p. 93.8 $94.7{ }^{\circ} \mathrm{C} ;{ }^{1} \mathrm{H}$ NMR (400 MHz, $\mathrm{CDCl}_{3}$ ) $\delta: 10.64$ (br s, $1 \mathrm{H}$, $\mathrm{NH}$ ), 8.54 (br s, $1 \mathrm{H}, \mathrm{NH}), 3.58 \sim 3.55(\mathrm{t}, J=5.2,6.8 \mathrm{~Hz}$, $\left.2 \mathrm{H}, \mathrm{NHCH}_{2}\right), 2.37\left(\mathrm{~s}, 3 \mathrm{H}, \mathrm{CCH}_{3}\right), 2.23\left(\mathrm{~s}, 3 \mathrm{H}, \mathrm{CCH}_{3}\right)$, $1.22 \sim 1.17\left(\mathrm{~m}, 1 \mathrm{H}, \mathrm{NHCH}_{2} \mathrm{CH}\right), 0.63 \sim 0.61(\mathrm{~d}, J=7.6$ $\left.\mathrm{Hz}, 2 \mathrm{H}, \mathrm{CHCH}_{2}\right), 0.36 \sim 0.34\left(\mathrm{~m}, 11 \mathrm{H}, \mathrm{CHCH}_{2}, \mathrm{SiCH}_{3}\right)$; ${ }^{13} \mathrm{C}$ NMR $\left(100 \mathrm{MHz}, \mathrm{CDCl}_{3}\right) \delta: 179.24,166.92,142.01$, $140.38,140.00,133.01,50.77,13.31,12.80,9.68,3.66$, 0.04. Anal. calcd for $\mathrm{C}_{15} \mathrm{H}_{24} \mathrm{~N}_{2} \mathrm{OS}_{2} \mathrm{Si}$ : C 52.90, H 7.10, N 8.23, S 18.83; found C 52.81, H 7.13, N 8.25, S 18.79.

1-(3-氯茮基)-3-(4,5-二甲基-2-三甲基硅基噻吩-3-甲 酰基)硫脲(6e)：淡黄色固体，收率 81\%. m.p. 101.9 $102.8{ }^{\circ} \mathrm{C} ;{ }^{1} \mathrm{H}$ NMR (400 MHz, $\mathrm{CDCl}_{3}$ ) $\delta: 10.95$ (br s, $1 \mathrm{H}$, $\mathrm{NH}), 8.64$ (br s, 1H, NH), 7.37 7.26 (m, 4H, Ar-H), $4.94 \sim 4.92\left(\mathrm{~d}, 2 \mathrm{H}, \mathrm{NHCH}_{2}\right), 2.37\left(\mathrm{~s}, 3 \mathrm{H}, \mathrm{CCH}_{3}\right), 2.23(\mathrm{~s}$, $\left.3 \mathrm{H}, \mathrm{CCH}_{3}\right), 0.36\left(\mathrm{~s}, 9 \mathrm{H}, \mathrm{SiCH}_{3}\right) ;{ }^{13} \mathrm{C} \mathrm{NMR}(100 \mathrm{MHz}$, $\left.\mathrm{CDCl}_{3}\right) \delta: 180.32,166.98,141.66,140.54,138.39,134.68$, $132.98,130.07,128.02,127.80,125.80,48.68,13.33$, 12.86, - 0.03. Anal. calcd for $\mathrm{C}_{18} \mathrm{H}_{23} \mathrm{ClN}_{2} \mathrm{OS}_{2} \mathrm{Si}$ : C 52.59, H 5.64, N 6.81, S 15.60; found C 52.48, H 5.61, N 6.83, S 15.53 .

3-(4,5-二甲基-2-三甲基硅基噻吩-3-甲酰基)-1,1-二 乙基硫脲(6f): 淡黄色固体，收率 71\%. m.p. 105.9 
$106.9{ }^{\circ} \mathrm{C} ;{ }^{1} \mathrm{H}$ NMR $\left(400 \mathrm{MHz}, \mathrm{CDCl}_{3}\right) \delta: 7.80($ br s, $1 \mathrm{H}$, $\mathrm{NH}), 4.01\left(\mathrm{~s}, 2 \mathrm{H}, \mathrm{CH}_{2}\right), 3.69\left(\mathrm{~s}, 2 \mathrm{H}, \mathrm{CH}_{2}\right), 2.36(\mathrm{~s}, 3 \mathrm{H}$, $\mathrm{CH}_{2}$ ), 2.25 (s, 3H, $\mathrm{CH}_{2}$ ), 1.35 (s, 6H, $\left.\mathrm{CH}_{3}\right), 0.35$ (s, 9H, $\left.\mathrm{SiCH}_{3}\right) ;{ }^{13} \mathrm{C}$ NMR $\left(100 \mathrm{MHz}, \mathrm{CDCl}_{3}\right) \delta: 178.72,163.25$, 142.95, 140.04, 139.84, 132.83, 47.95, 13.39, 13.31, 11.53, 0.17. Anal. calcd for $\mathrm{C}_{15} \mathrm{H}_{26} \mathrm{~N}_{2} \mathrm{OS}_{2} \mathrm{Si}$ : C 52.59, H 7.65, N 8.18, S 18.72; found C 52.38, H 7.67, N 8.15, S 18.68.

1-烯丙基-3-(4,5-二甲基-2-氯甲基二甲基硅基噻吩3-甲酰基)硫嫝 $(\mathbf{6 g}$ ): 淡黄色固体, 收率 77\%. m.p. 74.5 $75.7{ }^{\circ} \mathrm{C} ;{ }^{1} \mathrm{H}$ NMR (400 MHz, $\left.\mathrm{CDCl}_{3}\right) \delta: 10.63$ (br s, $1 \mathrm{H}$, $\mathrm{NH}), 8.65($ br s, $1 \mathrm{H}, \mathrm{NH}), 6.02 \sim 5.95\left(\mathrm{~m}, 1 \mathrm{H}, \mathrm{NCH}_{2}-\right.$ $\mathrm{CH}=), 5.35 \sim 5.27\left(\mathrm{dd}, J=18.8,10.4 \mathrm{~Hz}, 2 \mathrm{H},=\mathrm{CH}_{2}\right)$, $4.38 \sim 4.36\left(\mathrm{~m}, 2 \mathrm{H}, \mathrm{NHCH}_{2}\right), 3.09\left(\mathrm{~s}, 2 \mathrm{H}, \mathrm{SiCH}_{2} \mathrm{Cl}\right)$, 2.39(s, 3H, Ar- $\mathrm{CH}_{3}$ ), 2.29 (s, 3H, Ar- $\left.\mathrm{CH}_{3}\right), 0.45$ (s, 6H, $\left.\mathrm{Si}-\mathrm{CH}_{3}\right) ;{ }^{13} \mathrm{C} \mathrm{NMR}\left(100 \mathrm{MHz}, \mathrm{CDCl}_{3}\right) \delta: 180.11,166.39$, $142.68,142.44,138.07,132.94,132.27,118.05,48.37$, 30.81, 14.01, 13.67, -2.85 . Anal. calcd for $\mathrm{C}_{14} \mathrm{H}_{21} \mathrm{ClN}_{2}$ $\mathrm{OS}_{2} \mathrm{Si}$ : C 46.58, H 5.86, N 7.76, S 17.76; found C 46.60, H 5.88, N 7.73, S 17.71 .

1-烯丙基-3-(4,5-二甲基噻吩-3-甲酰基)硫腿(8a): 淡黄色固体, 收率 68\%. m.p. 99.9 100.8 ${ }^{\circ} \mathrm{C} ;{ }^{1} \mathrm{H}$ NMR $\left(400 \mathrm{MHz}, \mathrm{CDCl}_{3}\right) \delta: 10.70(\mathrm{br} \mathrm{s}, 1 \mathrm{H}, \mathrm{NH}), 8.84(\mathrm{br} \mathrm{s}, 1 \mathrm{H}$, $\mathrm{NH}), 7.60(\mathrm{~s}, 1 \mathrm{H}, \mathrm{ArH}), 6.00 \sim 5.93\left(\mathrm{~m}, 1 \mathrm{H}, \mathrm{NHCH}_{2} \mathrm{CH}=\right.$ $\left.\mathrm{CH}_{2}\right), 5.35 \sim 5.31(\mathrm{~d}, J=17.2 \mathrm{~Hz}, 1 \mathrm{H}, \mathrm{CH}=\mathrm{CHH}), 5.27 \sim$ $5.25(\mathrm{~d}, J=10.4 \mathrm{~Hz}, 1 \mathrm{H}, \mathrm{CH}=\mathrm{CHH}), 4.36 \sim 4.33(\mathrm{dd}, J=$ 5.6, $5.2 \mathrm{~Hz}, 2 \mathrm{H}, \mathrm{NHCH}_{2}$ ), 2.37 (s, 3H, $\mathrm{ArCH}_{3}$ ), 2.29 (s, $\left.3 \mathrm{H}, \mathrm{ArCH}_{3}\right) ;{ }^{13} \mathrm{C} \mathrm{NMR}\left(100 \mathrm{MHz}, \mathrm{CDCl}_{3}\right) \delta: 180.35$, $164.05,136.52,134.57,133.34,132.12,126.46,118.00$, 48.28, 13.50, 13.11. Anal. calcd for $\mathrm{C}_{11} \mathrm{H}_{14} \mathrm{~N}_{2} \mathrm{OS}_{2}$ : C 51.94, H 5.55, N 11.01, S 25.21; found C 51.78, H 5.58, N 10.97, S 25.24 .

1-(4,5-二甲基噻吩-3-甲酰基)-3-异丙基硫脲 (8b): 淡黄色固体, 收率 79\%. m.p. 118.8 119.1 ${ }^{\circ} \mathrm{C} ;{ }^{1} \mathrm{H}$ NMR $\left(400 \mathrm{MHz}, \mathrm{CDCl}_{3}\right) \delta: 10.49$ (br s, $\left.1 \mathrm{H}, \mathrm{NH}\right), 8.72$ (br s, $1 \mathrm{H}$, $\mathrm{NH}), 7.59$ (s, 1H, Ar-H), $4.58 \sim 4.50$ (m, 1H, NHCH), 2.38 (s, 3H, Ar- $\mathrm{CH}_{3}$ ), 2.29 (s, 3H, Ar- $\mathrm{CH}_{3}$ ), 1.36 1.34 (d, $J=$ $\left.6.8 \mathrm{~Hz}, 6 \mathrm{H}, \mathrm{CHCH}_{3}\right) ;{ }^{13} \mathrm{C}$ NMR $\left(100 \mathrm{MHz}, \mathrm{CDCl}_{3}\right) \delta$ : $178.74,164.08,136.40,134.67,133.26,126.39,48.00$, 21.94, 13.48, 13.09. Anal. calcd for $\mathrm{C}_{11} \mathrm{H}_{16} \mathrm{~N}_{2} \mathrm{OS}_{2}$ : C 51.53, H 6.29, N 10.93, S 25.01; found C 51.42, H 6.31, N 10.89, S 24.97 .

1-环丙基-3-(4,5-二甲基噻吩-3-甲酰基)硫脲(8c): 淡 黄色固体, 收率 $65 \%$. m.p. 127.1 128.1 ${ }^{\circ} \mathrm{C} ;{ }^{1} \mathrm{H}$ NMR $\left(400 \mathrm{MHz}, \mathrm{CDCl}_{3}\right) \delta: 10.56($ br s, $1 \mathrm{H}, \mathrm{NH}), 8.78(\mathrm{br} \mathrm{s}, 1 \mathrm{H}$, $\mathrm{NH}), 7.58$ (s, 1H, Ar-H), 3.23 3.19 (m, 1H, NHCH), 2.36 (s, 3H, Ar- $\mathrm{CH}_{3}$ ), $2.28\left(\mathrm{~s}, 3 \mathrm{H}, \mathrm{Ar}-\mathrm{CH}_{3}\right), 0.98 \sim 0.93(\mathrm{~m}, 2 \mathrm{H}$, H-CHСН-H), $0.78 \sim 0.774(\mathrm{~m}, 2 \mathrm{H}, \mathrm{H}-\mathrm{CHCH}-\mathrm{H}) ;{ }^{13} \mathrm{C}$ NMR $\left(100 \mathrm{MHz}, \mathrm{CDCl}_{3}\right) \delta: 181.67,164.04,136.50$, 134.58, 133.29, 126.41, 28.29, 13.49, 13.09, 7.42. Anal. calcd for $\mathrm{C}_{11} \mathrm{H}_{14} \mathrm{~N}_{2} \mathrm{OS}_{2}$ : C 51.94, H 5.55, N 11.01, S 25.21; found C 51.84, H 5.53, N 11.05, S 25.23.

1-环丙基甲基-3-(4,5-二甲基噻吩-3-甲酰基)硫脲 (8d): 淡黄色固体, 收率 79\%. m.p. 115.3 $116.1{ }^{\circ} \mathrm{C} ;{ }^{1} \mathrm{H}$ NMR (400 MHz, $\mathrm{CDCl}_{3}$ ) $\delta: 10.67$ (br s, $1 \mathrm{H}, \mathrm{NH}$ ), 8.75 (br s, $1 \mathrm{H}, \mathrm{NH}), 7.60(\mathrm{~s}, 1 \mathrm{H}, \mathrm{ArH}), 3.56 \sim 3.53$ (t, $J=5.2,6.8$ $\left.\mathrm{Hz}, 2 \mathrm{H}, \mathrm{NHCH}_{2}\right), 2.38$ (s, 3H, $\left.\mathrm{ArCH}_{3}\right), 2.31$ (s, 3H, $\left.\mathrm{ArCH}_{3}\right), 1.21 \sim 1.15\left(\mathrm{~m}, 1 \mathrm{H}, \mathrm{NHCH}_{2} \mathrm{CH}\right), 0.63 \sim 0.61(\mathrm{~d}$, $J=7.6 \mathrm{~Hz}, \mathrm{H}-\mathrm{CHCH}-\mathrm{H}), 0.34 \sim 0.33$ (d, $J=4.8 \mathrm{~Hz}$, $\mathrm{H}-\mathrm{CHCH}-\mathrm{H}) ;{ }^{13} \mathrm{C}$ NMR (100 MHz, $\left.\mathrm{CDCl}_{3}\right) \delta: 179.78$, $164.06,136.36,134.63,133.24,126.46,51.12,13.46$, 13.08, 9.84, 3.91. Anal. calcd for $\mathrm{C}_{12} \mathrm{H}_{16} \mathrm{~N}_{2} \mathrm{OS}_{2}$ : C 53.70, $\mathrm{H}$ 6.01, N, 10.44, S 23.89; found C 53.51, H 6.04, N 10.46, S 23.80 .

1-(3-氯苄基)-3-(4,5-二甲基噻吩-3-甲酰基)硫脲(8e): 淡黄色固体, 收率 80\%. m.p. 75.1 75.4 ${ }^{\circ} \mathrm{C} ;{ }^{1} \mathrm{H}$ NMR $\left(400 \mathrm{MHz}, \mathrm{CDCl}_{3}\right) \delta: 10.95$ (br s, $\left.1 \mathrm{H}, \mathrm{NH}\right), 8.88$ (br s, $1 \mathrm{H}$, $\mathrm{NH}), 7.62(\mathrm{~s}, 1 \mathrm{H}, \mathrm{ArH}), 7.37$ (s, 1H, ArH), $7.29 \sim 7.26(\mathrm{~m}$, $3 \mathrm{H}, \mathrm{ArH}$ ), $4.90 \sim 4.88$ (d, $\left.J=5.6 \mathrm{~Hz}, 2 \mathrm{H}, \mathrm{NHCH}_{2}\right), 2.37$ (s, $\left.3 \mathrm{H}, \mathrm{ArCH}_{3}\right), 2.28\left(\mathrm{~s}, 3 \mathrm{H}, \mathrm{ArCH}_{3}\right) ;{ }^{13} \mathrm{C}$ NMR $(100 \mathrm{MHz}$, $\left.\mathrm{CDCl}_{3}\right) \delta: 180.85,164.10,138.53,136.48,134.83,134.33$, $133.36,130.27,128.27,128.23,126.77,126.23,49.07$, 13.47, 13.11. Anal. calcd for $\mathrm{C}_{15} \mathrm{H}_{15} \mathrm{ClN}_{2} \mathrm{OS}_{2}$ : C 53.16, $\mathrm{H}$ 4.46, N 8.27, S 18.92; found C 53.10, H 4.43, N 8.31, S 18.88 .

3-(4,5-二甲基噻吩-3-甲酰基)-1,1-二乙基硫脲(8f): 淡黄色固体, 收率 74\%. m.p. 92.2 94.6 ${ }^{\circ} \mathrm{C} ;{ }^{1} \mathrm{H}$ NMR $\left(400 \mathrm{MHz}, \mathrm{CDCl}_{3}\right) \delta: 8.07$ (br s, 1H, NH), $7.56(\mathrm{~s}, 1 \mathrm{H}$, ArH), 4.01 (br s, 2H, NHCH $), 3.61$ (br s, 2H, $\mathrm{NHCH}_{2}$ ), 2.36 (s, $3 \mathrm{H}, \mathrm{ArCH}_{3}$ ), 2.30 (s, $3 \mathrm{H}, \mathrm{ArCH}_{3}$ ), 1.30 (br s, $3 \mathrm{H}$, $\mathrm{CH}_{2} \mathrm{CH}_{3}$ ), 1.26 (br s, 3H, $\mathrm{CH}_{2} \mathrm{CH}_{3}$ ); ${ }^{13} \mathrm{C}$ NMR (100 MHz, $\left.\mathrm{CDCl}_{3}\right) \delta: 179.37,161.30,135.67,135.20,133.62,126.34$, $48.06,47.80,13.40,13.12,11.76$. Anal. calcd for $\mathrm{C}_{12} \mathrm{H}_{18} \mathrm{~N}_{2} \mathrm{OS}_{2}$ : C 53.30, H 6.71, N 10.36, S 23.72; found C 53.25, H 6.69, N 10.31, S 23.65.

\section{4 化合物的生物活性测试}

化合物的生物活性测试采用平皿测定菌丝生长速 率法. 首先, 取新鲜土豆去皮后称取 $200 \mathrm{~g}$, 切成小块后 加入 $1 \mathrm{~L}$ 蒸馏水. 煮沸 $20 \mathrm{~min}$ 后用纱布将土豆滤出, 向 滤液中加入 $20 \mathrm{~g}$ 葡萄糖和 $15 \mathrm{~g}$ 琼脂, 待完全溶解后用蒸 馏水定容至 $1 \mathrm{~L}$, 在 $121{ }^{\circ} \mathrm{C}$ 下湿热灭菌 $20 \mathrm{~min}$, 制得马 
铃薯、葡萄糖、琼脂(PDA)培养基培养基.

然后, 吸取适当浓度的供试样品 $1 \mathrm{~mL}$ 丙酮溶液和 熔化并冷却至 $50{ }^{\circ} \mathrm{C}$ 左右的 $\mathrm{PDA}$ 培养基 $9 \mathrm{~mL}$ 倒入无菌 培养血中, 混合均匀后冷却至室温. 将在 PDA 平板上培 养 $7 \mathrm{~d}$ 的小麦全蚀病病原菌用直径 $5 \mathrm{~mm}$ 的打孔器制作 成菌碟, 并将菌碟接种在含药的 PDA 平板中央, 以不含 药的平板培养基为对照. 每个药液浓度设 4 个重复, 在 $20 \sim 25{ }^{\circ} \mathrm{C}$ 培养箱中避光培养 $6 \mathrm{~d}$, 调查菌落直径, 计算 相对抑制率. 相对抑制率 $=$ (空白对照菌落直径一药剂 处理菌落直径)/空白对照菌落直径 $\times 100 \%$. 水稻纹枯病 活性测试采用相似的方法.

\section{References}

[1] Yan, Z. K.; Song, B. A.; Yang, X.; Hu, D. Y.; Yang, S.; Jin, L. H.; Chen, G. M. Agrochemicals 2008, 47, 706 (in Chinese). (间志坤，宋宝安，杨璇，胡德禹，杨松，金林红，陈广明，农药， 2008, 47, 706.)

[2] Ishaaya, I.; Mendelson, Z.; Horowitz, A. R. Phytoparasitica 1993, $21,199$.

[3] Sun, Z. H.; Huang, W.; Gong, Y. Y.; Lan, J.; Liu, X. H.; Weng, J. Q.; Li, Y. S.; Tan, C. X. Chin. J. Org. Chem. 2013, 33, 2612 (in Chinese).

(孙召慧, 黄伟, 贡云芸, 蓝健, 刘幸海, 翁建全, 李永曙, 谭成 侠，有机化学, 2013, 33, 2612.)

[4] Miao, H. J.; Zhang, J. W.; Yuan, H. Z.; Li, Y.; Xu, Y.; Li, H.; Yang, X. L.; Ling, Y. Chin. J. Org. Chem. 2012, 32, 915 (in Chinese). (苗宏健, 张继伟, 袁会珠, 李映, 徐炎, 李慧, 杨新玲, 凌云, 有机化学, 2012, 32, 915.)

[5] Saeed, A.; Khan, M. S.; Rafique, H.; Shahid, M.; Iqbal, J. Bioorg. Chem. 2014, 52, 1 .

[6] Williams, D. J.; Dimmic, M. W.; Haakenson, W. P. J.; Wideman, A.; Shortt, B. J.; Cheeseright, T.; Crawford, M. J. WO 2009023721, 2009 [Chem. Abstr. 2009, 150, 230986].

[7] Dolman, H.; Kuipers, J. EP 234622, 1987 [Chem. Abstr. 1988, 109, 110245].
[8] Kim, D. S.; Chun, S. J.; Jeon, J. J.; Lee, S. W.; Joe, G. H. Pest Manage. Sci. 2004, 60, 1007

[9] Phillion, D.; Wong, S. C.; Shortt, B. US 5486621, 1996 [Chem. Abstr. 1996, 124, 253325].

[10] Yoshikawa, Y.; Tomitani, K.; Katsuta, H.; Kawashima, H.; Takahashi, T.; Inami, S.; Yanase, Y.; Takashi, A.; Shimotori, H.; Tomura, N. JP 09301974, 1997 [Chem. Abstr. 1997, 128, 22908].

[11] Xie, G. Y.; Jin, W. B.; Zhao, Y. Q.; Cheng, Y. N.; Sun, B. J.; Sun, S. J.; Wang, M. Z.; Wei D. D.; Li, H. L. Chin. J. Org. Chem. 2014, 34, 1124 (in Chinese).

(谢桂英，靳文波，赵艳芹，程绎南，孙炳剑，孙淑君，汪梅子， 位丹丹, 李洪连, 有机化学, 2014, 34, 1124.)

[12] Jin, W. B.; Xie, G. Y.; Sun, S. J.; Zhao, Y. Q.; Cheng, Y. N.; Sun, B. J.; Li, H. L. Chin. J. Org. Chem. 2014, 34, 2376 (in Chinese). (靳文波，谢桂英，孙淑君，赵艳芹，程绎南，孙炳剑，李洪连， 有机化学, 2014, 34, 2376.)

[13] Fevig, T. L.; Pillips, W. G.; Lau, P. H. J. Org. Chem. 2001, 66, 2493.

[14] Bures, E.; Spinazze, P. G.; Beese, G.; Hunt, I. R.; Rogers, C.; Keay, B. A. J. Org. Chem. 1997, 62, 8741.

[15] Lu, B.; Falck, J. R. Angew. Chem., Int. Ed. 2008, 47, 7508.

[16] Kuznetsov, A.; Onishi, Y.; Inamoto, Y.; Gevorgyan, V. Org. Lett. 2013, 15, 2498.

[17] Ihara, H.; Koyanagi, M.; Suginome, M. Org. Lett., 2011, 13, 2662.

[18] Cao, H.; Zhan, H. Y.; Wu, J. Y.; Zhong, H. P.; Lin, Y. G.; Zhang, H. Eur. J. Org. Chem. 2012, 2318.

[19] Cao, H.; Jiang, H. F.; Zhou, X. S.; Qi, C. R.; Lin, Y. G.; Wu, J. Y.; Liang, Q. M. Green Chem. 2012, 14, 2710.

[20] Baharfar, R.; Baghbaniana, S. M.; Hossein, N. R.; Bijanzadeh, H. R. Lett. Org. Chem. 2007, 4, 567.

[21] Reddy, L. R.; Saravanan, P.; Corey, E. J. J. Am. Chem. Soc. 2004, 126,6230

[22] Downey, C. W.; Craciun, S.; Neferu, A. M.; Vivelo, C. A.; Mueller, C. J.; Southall, B. C.; Corsi, S.; Etchill, E. W.; Sault, R. J. Tetrahedron Lett. 2012, 53, 5763

[23] Bohlmann, F.; Bresinsky, E. Chem. Ber. 1964, 97, 2109.

[24] Sun, B. J.; Yuan, H. X.; Xing, X. P.; Li, H. L. J. Triticeae Crops 2008, 28, 709 (in Chinese).

(孙炳剑，袁虹霞，邢小萍，李洪连，麦类作物学报， 2008，28， 709.)

(Li, L.; Fan, Y.) 